\title{
Continuous intranigral infusion is not associated with observable behavioral deficits or marked pathology: a preclinical safety study
}

\author{
Richard Grondin, PhD, ${ }^{1}$ Yi Ai, MD, ${ }^{1}$ Peter A. Hardy, PhD, ${ }^{2}$ Mark T. Butt, DVM, DACVP, ${ }^{3}$ \\ Brian D. Nelson, BS, ${ }^{4}$ Jack D. Lemmon, PhD, ${ }^{4}$ David Bumcrot, PhD, ${ }^{5}$ Don M. Gash, PhD, ${ }^{1}$ \\ Greg A. Gerhardt, PhD, ${ }^{1}$ and Zhiming Zhang, MD'
}

\begin{abstract}
Departments of ${ }^{1}$ Anatomy and Neurobiology and ${ }^{2}$ Radiology, University of Kentucky Medical Center, Lexington, Kentucky; ${ }^{3}$ Tox Path Specialists LLC, Frederick, Maryland; " Medtronic Inc., Minneapolis, Minnesota; and ${ }^{5}$ Alnylam Pharmaceuticals Inc., Cambridge, Massachusetts
\end{abstract}

\begin{abstract}
OBJECTIVE A better understanding of the effects of chronically delivering compounds to the substantia nigra and nearby areas is important for the development of new therapeutic approaches to treat alpha-synucleinopathies, like Parkinson's disease. Whether chronic intranigral delivery of an infusate could be achieved without causing motor dysfunction or marked pathology remains unclear. The authors evaluated the tolerability of continuously delivering an infusate directly into the rhesus monkey substantia nigra via a programmable pump coupled to a novel intraparenchymal needle-tip catheter surgically implanted using MRI-guided techniques.
\end{abstract}

METHODS The MRI contrast agent gadopentetate dimeglumine (Magnevist, $5 \mathrm{mM}$ ) was used to noninvasively evaluate catheter patency and infusion volume associated with 2 flow rates sequentially tested in each of 3 animals: $0.1 \mu \mathrm{l} / \mathrm{min}$ for 14 days into the right substantia nigra and $0.1 \mu \mathrm{l} / \mathrm{min}$ for 7 days plus $0.2 \mu \mathrm{l} / \mathrm{min}$ for an additional 7 days into the left substantia nigra. Flow rate tolerability was assessed via clinical observations and a microscopic examination of the striatum and midbrain regions.

RESULTS Evaluation of postsurgical MRI indicated that all 6 catheters remained patent throughout the study and that the volume of distribution achieved in the left midbrain region at a rate of up to $0.2 \mu \mathrm{l} / \mathrm{min}\left(2052 \pm 168 \mathrm{~mm}^{3}\right)$ was greater than that achieved in the right midbrain region at a constant rate of $0.1 \mu \mathrm{l} / \mathrm{min}\left(1225 \pm 273 \mathrm{~mm}^{3}\right)$ by nearly 2 -fold. Both flow rates provided sufficient infusate coverage of the rhesus (and possibly the human) midbrain region. There were no indications of observable deficits in behavior. Histopathological evaluations confirmed that all catheter tips were placed in or near the pars compacta region of the substantia nigra in all animals. There was no evidence of infection at any of the 6 catheter sites. Mild to moderate microglial reactions were observed at most catheter track sites and were comparable between the 2 infusion rates. Finally, there was neither observable decrease of tyrosine hydroxylase staining in the striatum nor detectable necrosis of neurons in the pars compacta region of the substantia nigra in any of the animals.

CONCLUSIONS The data from this study support the feasibility of using a pump-and-catheter system for chronic intranigral infusion and lay the foundation for using this approach to treat Parkinson's disease or other related degenerative diseases that would benefit from targeted drug delivery to the substantia nigra or to other brainstem regions.

https://thejns.org/doi/abs/10.3171/2016.2.JNS152295

KEY WORDS Parkinson's disease; MRI; segmentation; Magnevist; gadopentetate dimeglumine; rhesus; substantia nigra

$\mathrm{P}$ ARKINSON's disease (PD) is a progressive, neurodegenerative alpha-synucleinopathy clinically characterized by motor and nonmotor deficits such as changes in blood pressure, bowel/bladder function, and loss of sense of smell. The histopathological hallmarks of
PD include dopamine cell loss in the substantia nigra pars compacta and the formation of alpha-synuclein-rich Lewy bodies and Lewy neurites in surviving neurons. ${ }^{21}$ While the motor symptoms in affected individuals are primarily due to dopamine cell loss in the substantia nigra pars

ABBREVIATIONS GDNF = glial cell-line derived neurotrophic factor; HLA = human leukocyte antigen; IM = intramuscularly; PB = phosphate buffer; PD = Parkinson's disease; siRNA = small interfering RNA; TH = tyrosine hydroxylase.

SUBMITTED September 30, 2015. ACCEPTED February 19, 2016.

INCLUDE WHEN CITING Published online May 27, 2016; DOI: 10.3171/2016.2.JNS152295. 
compacta, the nonmotor features arise from pathology in the olfactory bulb/tract and neuronal loss in extranigral brainstem areas, including the dorsal motor nucleus of vagus nerve, the raphe system, and the coeruleus-subcoeruleus complex. ${ }^{6}$ More than 40 years after the approval of levodopa for therapeutic use in PD patients, ${ }^{14}$ treatments of motor and nonmotor complications still remain largely symptomatic, with most of that effort focusing on the nigrostriatal pathway.

A few clinical studies have sought to use trophic factor administration to potentially slow or halt disease progression. For example, direct delivery to the striatum of glial cell-line derived neurotrophic factor (GDNF) has been safely achieved in PD patients by using a pump-andcatheter system..$^{10,12,19}$ Although significant improvements in motor function have been reported in some of these GDNF-treated patients, ${ }^{8,19}$ in others no such improvement has been seen after GDNF treatment. ${ }^{12}$ The lack of clinical efficacy in these patients may have been due to insufficient tissue distribution of trophic factor in the brain parenchyma. ${ }^{18}$ Another possibility lies with the site of drug administration itself, namely the striatum, requiring the trophic factors to be retrogradely transported back to the midbrain dopamine neurons. Postmortem studies suggest that axonal transport may reduce and/or delay clinical benefits resulting from intrastriatal trophic factor administration., ${ }^{4,5}$ One potential approach that could be used to help overcome the limitations related to axonal transport would be via direct drug delivery to the substantia nigra region.

Although the substantia nigra is a relatively small structure located near critical neuronal and vascular structures, acute delivery of viral vectors encoding for the trophic factor neurturin directly into the substantia nigra has been shown to be safe in both nonhuman primates and PD patients. ${ }^{2,3}$ Alternatively, programmable pumps coupled to intraparenchymal catheters placed directly into the midbrain substantia nigra could be used for the chronic administration of a variety of therapeutic agents to treat PD, including trophic factors and small interfering RNAs (siRNAs) designed to reduce the expression of proteins shown to play a role in the pathogenesis of PD, like alpha-synuclein. ${ }^{13,15}$ Also, targeted drug delivery to other extranigral brainstem areas could potentially benefit a host of disorders for which there is no cure, including multiple-system atrophy.

Several studies have safely infused therapeutic molecules to brain regions/space like the striatum and lateral ventricles in both animals and humans.,10-12,20 Whether chronic infusion of trophic factors or other potential disease-modifying molecules to the substantia nigra or other brainstem areas could be safely achieved using a pumpand-catheter system without causing motor dysfunction or marked pathology remains poorly studied. In addition, numerous technical issues associated with nigral infusions, including the delivery rate needed to achieve adequate coverage of the substantia nigra region, are poorly understood. Thus, the present study was conducted to evaluate the tolerability of continuously delivering an infusate directly into the adult rhesus macaque midbrain using an investigational catheter designed to create minimal tissue damage and minimize backflow. An MRI contrast agent was used to noninvasively evaluate catheter patency and infusion volume associated with 2 flow rates sequentially tested in the right and left substantia nigra of each animal. Each implant cycle lasted 14 days. Flow-rate tolerability was assessed via clinical observations, body weight measurements, and a microscopic examination of the striatum and midbrain regions. Our data lay the foundation for locally delivering therapeutic compounds via a pump and indwelling catheter system to treat patients who would benefit from direct drug delivery into the substantia nigra or other brainstem areas.

\section{Methods}

\section{Animals}

Adult, female rhesus macaques (Macaca mulatta; $\mathrm{n}=$ 3 ) ranging in age from 7 to 11 years and weighing 4.5-8.0 $\mathrm{kg}$ were obtained from commercial vendors and housed in the vivarium at the University of Kentucky Division of Laboratory Animal Resources, which is fully accredited by the Association for Assessment and Accreditation of Laboratory Animal Care International. All procedures were carried out in accordance with the standards detailed in the Guide for the Care and Use of Laboratory Animals and approved by the University of Kentucky Institutional Animal Care and Use Committee.? In addition, experienced veterinarians working closely with skilled laboratory technicians and animal caretakers supervised all animal care. The animals were housed in the same room in individual living modules, to help preserve the integrity of the implanted delivery system. They were maintained on a 12-hour light/dark cycle in temperature- and humiditycontrolled rooms. Each animal was given access to an activity module several hours per week and provided toys as well as other devices for enrichment. Except on days where procedures requiring anesthesia were performed, the animals were fed a standard commercial diet of nonhuman primate chow (Harlan 2050 Teklad Global 20\% Protein Primate Diet, Envigo), supplemented with fresh fruit or vegetables. Municipal tap water, purified by reverse osmosis, was available ad libitum via an automated watering system.

\section{Study Design}

The commercially available MRI contrast agent gadopentetate dimeglumine (Magnevist, Bayer Pharmaceuticals Inc.) was used to noninvasively evaluate catheter patency and infusion volume associated with 2 flow rates sequentially tested in each animal: $0.1 \mu \mathrm{l} / \mathrm{min}$ for 14 days in the right substantia nigra, and $0.1 \mu \mathrm{l} / \mathrm{min}$ for 7 days plus $0.2 \mu \mathrm{l} / \mathrm{min}$ for an additional 7 days in the left substantia nigra. Gadopentetate dimeglumine $(5 \mathrm{mM})$ was infused via an investigational needle-tip catheter and cranial anchor system coupled to an implanted programmable pump, as described below. A single pump implanted during the first surgery was used for both catheters. Briefly, a single catheter was first placed in the right substantia nigra of each animal and the implanted pump programmed at a rate of $0.1 \mu \mathrm{l} / \mathrm{min}$ for the first 7 days during the postoperative recovery period. Infusion continued at the same rate for 7 more days, for a total of 14 days. Then, the first 
anchor/catheter assembly was removed from the right substantia nigra and a second anchor/catheter assembly was positioned in the left substantia nigra. The pump was programmed at a rate of $0.1 \mu \mathrm{l} / \mathrm{min}$ during the 7 -day postsurgery recovery period, at which point the flow rate was doubled to $0.2 \mu \mathrm{l} / \mathrm{min}$ for an additional 7 days and the animals killed. MR images were acquired at the end of each 14-day infusion period, as described below. Gadopentetate dimeglumine concentration and flow rates for intranigral infusion were selected based on our prior nonhuman primate infusion studies conducted in the larger putamen at rates ranging from $0.1 \mu \mathrm{l} / \mathrm{min}$ to $0.5 \mu \mathrm{l} / \mathrm{min} .{ }^{9,20}$

\section{Delivery System}

The delivery system was provided by Medtronic Inc. and consisted of a 20-ml capacity, titanium-encased SynchroMed II pump (model 8637-20) surgically implanted in the subcutaneous layer of the lateral abdominal region and connected subcutaneously via tubing to an experimental anchor attached to the skull with 2 screws. ${ }^{11}$ The anchor, in turn, was connected to an experimental catheter stereotactically inserted into the substantia nigra. The intraparenchymal catheter is made of 55D urethane $(0.60$ $\mathrm{mm}$ inner diameter; $1.0 \mathrm{~mm}$ outer diameter) and terminates as a closed-off needle tip. Unlike other flexible intraparenchymal catheters, ${ }^{1,10-12,18,19}$ the redesigned tip of the catheter is composed of a small-diameter platinum iridium needle $(0.10-\mathrm{mm}$ inner diameter; 0.20 - $\mathrm{mm}$ outer diameter) and 2 lateral side ports $(0.10 \mathrm{~mm}$ in diameter $)$ designed to prevent tissue coring and to lower the peak pressure during initial infusion. The side ports are positioned $180^{\circ}$ from each other at $0.5 \mathrm{~mm}$ from the most distal end of the closed-off tip (Fig. 1). As illustrated in Fig. 1, the $15-\mathrm{mm}$-long needle extends $5 \mathrm{~mm}$ below the catheter step, a feature designed to minimize backflow. The rate and timing of delivery was noninvasively adjusted using the Medtronic N'Vision pump programmer (model 8840).

\section{MRI-Guided Stereotactic Catheter Placement}

Animals were sedated with ketamine $(10-20 \mathrm{mg} / \mathrm{kg}$ intramuscularly [IM]) admixed with atropine (0.02-0.04 $\mathrm{mg} / \mathrm{kg} \mathrm{IM}$ ) and intubated for administration of isoflurane gas anesthesia $(1 \%-3 \%)$ in $\mathrm{O}_{2}$ to effect. The anesthetized animals were placed in an MRI-compatible stereotaxic head frame in a ventral-lateral position for subsequent MRI acquisition on a Siemens 3T Tim Trio MR imager. MRI coordinates used to guide catheter placement were derived from T1-weighted coronal brain images acquired immediately prior to surgery for each animal in the following ranges: $8-12 \mathrm{~mm}$ at the anteroposterior coordinate (i.e., distance from interaural line to the target), $3.5-4.5 \mathrm{~mm}$ at the lateral coordinate (i.e., distance from the sagittal sinus/ third ventricle to the target), and $33-35 \mathrm{~mm}$ at the vertical coordinate (i.e., distance from the brain surface to the target).

Upon transportation of the animals from the MRI scanner to the experimental surgery suite, the abdominal and cranial surgical sites were prepared using antiseptic techniques. An incision was made through the scalp under

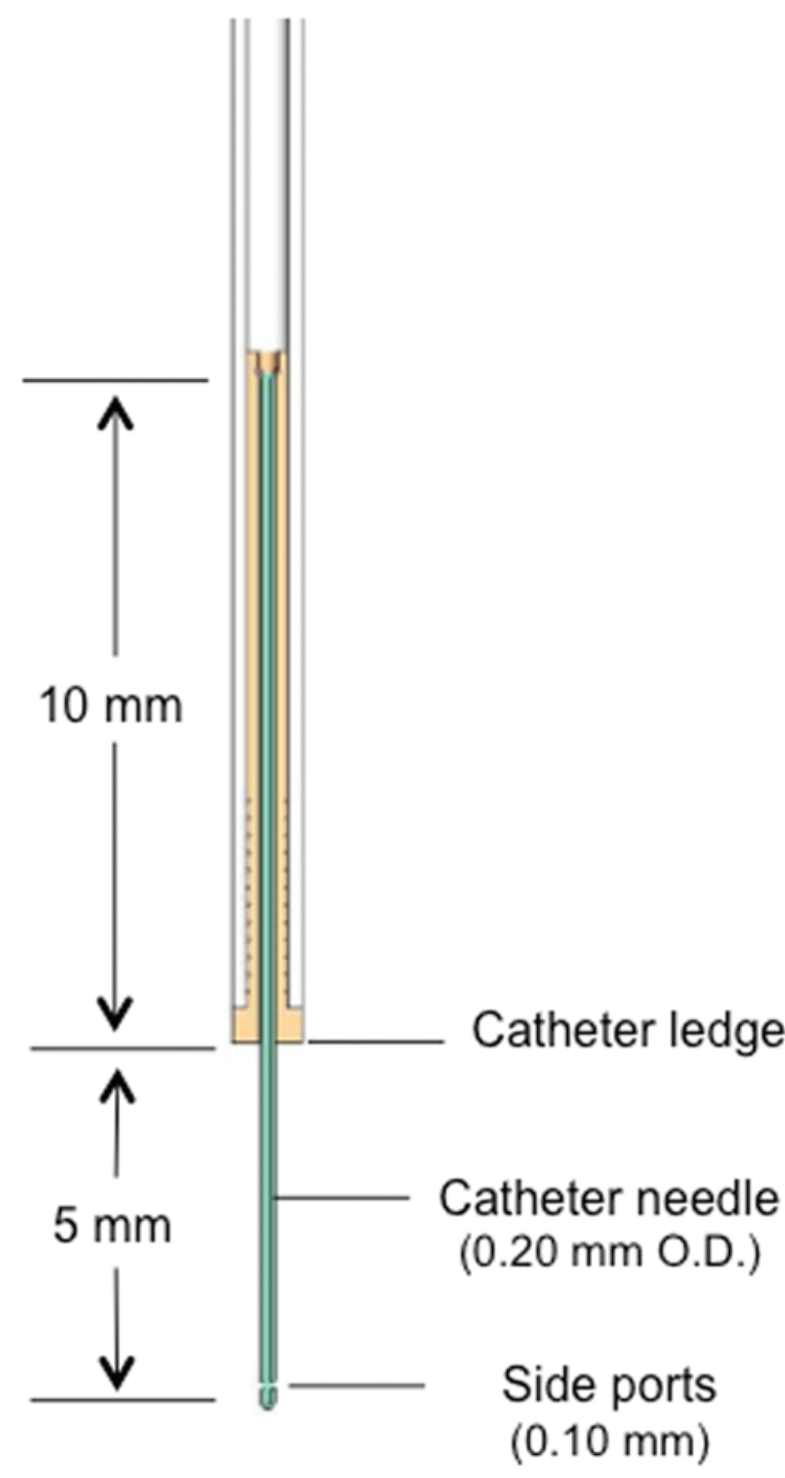

FIG. 1. Catheter tip design. The tip of the intraparenchymal catheter is composed of a platinum iridium needle $(0.20-\mathrm{mm}$ O.D.) with 2 lateral side ports of $0.10 \mathrm{~mm}$ in diameter positioned $180^{\circ}$ from each other at $0.5 \mathrm{~mm}$ from the most distal end of the closed-off tip. The 15-mm-long needle extends $5 \mathrm{~mm}$ below the catheter step, a feature designed to minimize backflow. O.D. = outer diameter. Figure is available in color online only.

sterile field conditions, and the skin and muscles overlying the skull were reflected. Using MRI-guided procedures, a small bur hole was drilled in the skull directly over the target area, and the overlying meninges were punctured to expose the surface of the brain. Using a micromanipulator, a cannula containing an obturator was lowered into the brain at a rate of approximately $1 \mathrm{~mm}$ per minute to a depth of about $7 \mathrm{~mm}$ above the substantia nigra. Next, the obturator was removed from the cannula and the intraparenchymal catheter containing a hollow stylet was lowered until its needle tip was at a vertical depth corresponding to the planned target (i.e., $33-35 \mathrm{~mm}$ from the surface of the brain). Throughout this procedure, sterile saline was infused through the hollow stylet and catheter at a rate 
of $1.0 \mu \mathrm{l} / \mathrm{min}$ to maintain catheter patency. ${ }^{9}$ The catheter was secured at the skull using a cranial anchor. In parallel to catheter placement, a gadopentetate dimeglumine-filled pump was surgically implanted into the lateral abdominal region and connected to a gadopentetate dimeglumineprimed tubing, which was tunneled under the skin and also attached to the anchor. After completion of the procedures, the incisions were sutured over the exposed areas.

Heat support and fluid replacement were provided during the surgical procedures and vital signs were monitored. Buprenorphine $(0.01 \mathrm{mg} / \mathrm{kg})$ was administered before beginning the surgical procedures and again approximately 6 hours later. In addition, a pediatric fentanyl patch $(25 \mu \mathrm{g} / \mathrm{hr}$ for animals weighing less than $7 \mathrm{~kg}$ or 50 $\mu \mathrm{g} / \mathrm{hr}$ for animals weighing more than $7 \mathrm{~kg}$ ) was placed on a shaved area between the animal's shoulder blades prior to beginning the surgical procedures, to provide analgesia for approximately 3 days.

\section{Catheter Patency and Volume of Distribution in the Brain Parenchyma}

Catheter placement as well as initial catheter patency was verified using noninvasive neuroimaging performed within approximately 30 minutes of postsurgical implantation. Flow rate parameters were as follows: 1) $0.1 \mu \mathrm{l} / \mathrm{min}$ for 14 days in the right substantia nigra, and 2) $0.1 \mu \mathrm{l} / \mathrm{min}$ for the first 7 days plus $0.2 \mu \mathrm{l} / \mathrm{min}$ for another 7 days in the left substantia nigra.

The distribution volume of gadopentetate dimeglumine detectable in the midbrain region was measured from MR images acquired in anesthetized animals after a 14-day infusion period for each catheter site. Volumetric values are expressed in cubic millimeters. Heat support was provided during the imaging sessions and vital signs were monitored using a telemetry-operated physiological monitor (PRECESS, Invivo Corp.). Images were acquired on a 3T Siemens Tim Trio MR imager using a custom-developed, phased-array surface coil to enhance the signal acquisition from the brain of the animal. The image acquisition consisted of a pair of 3D, T1-weighted fast, low, angle shot images covering the entire brain. The 3D images had identical resolution: $0.66 \mathrm{~mm} \times 0.66 \mathrm{~mm}$ in-plane and 1.0 $\mathrm{mm}$ through plane and identical acquisition parameters, except different excitation angles $\alpha=15^{\circ}$ and $75^{\circ}$. These angles were designed according to the method of Deoni and colleagues ${ }^{8}$ to provide an accurate measurement of low T1 values. From the pair of 3D images, a single 3D image set corresponding to $1 / \mathrm{T} 1$ was calculated. Because $1 / \mathrm{T} 1$ is proportional to the concentration of gadopentetate dimeglumine, the image intensity in this calculated image set can be used for estimating the distribution of gadopentetate dimeglumine. A computer program was developed to identify the area on each image corresponding to the infusion of gadopentetate dimeglumine. The operator selected a point near the tip of the catheter where gadopentetate dimeglumine had accumulated. The program automatically included all contiguous pixels above background level. The total volume was determined by summing the number of pixels included in the segmentation process and multiplying by the volume of 1 pixel (i.e., $0.444 \mathrm{~mm}^{3}$ ).

\section{Clinical Observations and Body Weight Measurements}

Daily cage-side observations for morbidity and mortality were performed on all animals beginning at least 3 days prior to surgery and continuing through the day of necropsy. Cage-side observations included evaluations of the incision site for redness, swelling, and discharge, and for any other abnormal clinical signs such as vomiting, seizures, bradykinesia, akinesia, and/or ataxia. Also, individual body weights were measured for all animals before surgery and weekly thereafter through the day of necropsy.

\section{Tissue Processing and Histopathological Analysis}

At the end of the study, the animals were killed by barbiturate overdose (greater than $50 \mathrm{mg} / \mathrm{kg}$ ) and transcardially perfused with heparinized ice-cold $0.1 \mathrm{M}$ phosphate buffer (PB) followed by $4 \%$ methanol-free formaldehyde (made from paraformaldehyde powder) in $0.1 \mathrm{M} \mathrm{PB}(\mathrm{pH}$ 7.4) for head fixation. The whole brain was quickly removed, postfixed in the same fixative for a maximum of 48 hours, and cryoprotected. Cryoprotected tissue at the level of the substantia nigra was sectioned frozen at 40 microns. Then, 1 in every 24 sections was processed for $\mathrm{H} \& \mathrm{E}$ (general morphology), Nissl (specific for neurons), and Fluoro-Jade B (EMD Millipore Corp.) staining to visualize neuronal necrosis. Using procedures described previously, ${ }^{1}$ adjacent sections were processed by immunohistochemistry for glial fibrillary acidic protein (1:5000, Catalog No. MAB360, Chemicon) and human leukocyte antigen-antigen D related (HLA-DR 1:200, Catalog No. 347360 , Becton Dickinson Biosciences) to evaluate astrocytosis and activated microglia, respectively.

Histopathological examination of preserved tissues was performed by a board-certified veterinary pathologist with specific expertise in evaluating the nervous system. For each specific stain, histopathological evaluations were conducted on full coronal sections, including 3-4 midbrain sections that spanned the infusion site. In addition, 1 section from the striatum stained for tyrosine hydroxylase (TH; 1:2000, Catalog No. MAB318, Millipore) was included in the analysis to further assess potential substantia nigra injury. As further described in Table 1, microscopic findings were graded according to a 5-point semiquantitative grading scale in order of increasing severity: Grade 1 (slight), Grade 2 (minimal), Grade 3 (mild), Grade 4 (moderate), and Grade 5 (severe). The various grades considered the relative degree of tissue involvement such that a notable focal response may be graded similarly to a more diffuse but less intense response.

\section{Statistical Analysis}

Data are presented as mean \pm SEM values. Body weight measurements associated with each flow rate were analyzed for significant differences over time using a 1-way analysis of variance for repeated measures. A Dunnett's test was used for post hoc comparisons, when applicable. Infusion volume measurements associated with each flow rate were analyzed for significant differences between the right and left hemisphere using a 1-tailed, unpaired t-test. A p value $<0.05$ was considered significant in all analyses. 
TABLE 1. Semiquantitative scale for microscopic evaluations

\begin{tabular}{|c|c|c|}
\hline Grade & Severity & Explanation \\
\hline 1 & Slight & $\begin{array}{l}\text { A microscopic change that barely exceeds normal limits or what would be considered to be the normal condition. Typically, these } \\
\text { are microscopic changes that are sporadic, focal, \& of no biological significance to the function or structure of the tissue. Micro- } \\
\text { scopic changes graded as "1" are not readily apparent in the tissue. Generally, a slight change affects }<1 \% \text { of the total tissue } \\
\text { that might be possibly affected. }\end{array}$ \\
\hline 2 & Minimal & $\begin{array}{l}\text { A microscopic change that is more readily apparent in the tissue than a slight (Grade } 1 \text { ) change but is still unlikely to produce any } \\
\text { structural or functional impairment. Typically, a grade of minimal indicates the microscopic change was present in one or a few } \\
\text { foci. Generally, a microscopic change graded as minimal affects }<5 \% \text { of the total tissue that might be possibly affected. }\end{array}$ \\
\hline 3 & Mild & $\begin{array}{l}\text { A microscopic change that is readily observed in the section but still of limited severity \& unlikely to have any biologically signifi- } \\
\text { cant effect on overall structure or function of the tissue. Mild changes may be focal, multifocal, or even diffuse. }\end{array}$ \\
\hline 4 & Moderate & $\begin{array}{l}\text { A microscopic change that is a prominent, conspicuous, \& easily identified feature of the tissue but is still not present at what } \\
\text { would be considered to be the maximum possible effect. A moderate change would reasonably be expected to have some } \\
\text { effect on the structure \&/or function of a tissue, although the correlating functional change may or may not be apparent. }\end{array}$ \\
\hline 5 & Severe & $\begin{array}{l}\text { A microscopic change that is a prominent, conspicuous, \& easily identified feature of the tissue, is present at a maximum sever- } \\
\text { ity (affects the majority of the tissue \&/or is very pronounced in one or more foci), \&/or is present at a severity that would be } \\
\text { expected to have a prominent effect on the structure \&/or function of a tissue, although the correlating functional change may } \\
\text { or may not be apparent. }\end{array}$ \\
\hline
\end{tabular}

\section{Results}

\section{Clinical Observations and Body Weight Measurements}

All animals recovered after catheter placement without clinically observable complications. There was no early mortality observed during the course of the study and all 3 animals survived until the scheduled termination of the study. There were no signs of morbidity or abnormal clinical observations such as infection of the surgical sites, vomiting, seizures, bradykinesia, akinesia, and/or ataxia noted in any of the animals examined during the course of the study. Other than a transient postoperative reduction in food consumption following each catheter placement, there were no abnormal changes in food intake (data not shown). Compared with presurgical baseline values, a slight but statistically significant $5.2 \% \pm 0.6 \%$ decline in body weight values was measured over time while infusing into the right midbrain at a maximum rate of $0.1 \mu \mathrm{l} /$ min (Fig. 2 upper). A nonsignificant $2.4 \% \pm 2.2 \%$ decline in body weight values was recorded over the same period of time while infusing into the left midbrain at a maximum rate of $0.2 \mu \mathrm{l} / \mathrm{min}$ (Fig. 2 lower).

\section{Catheter Patency and Volume of Distribution in the Brain Parenchyma}

All 3 animals underwent 2 sequential catheter implantations with 1 catheter inserted into the right substantia nigra and the other into the left substantia nigra, 2 weeks later. Examination of MR images taken immediately postsurgery indicated that all 6 intranigral catheters ( 2 per animal) were patent, as evidenced by the presence of a high contrast signal from the infusate in the brain tissue surrounding the catheter tip. Subsequent evaluations of MR images acquired after catheter placement into either the right or the left substantia nigra also indicated the presence of the infusate in the midbrain region at each catheter site in all 3 animals.

As illustrated in Fig. 3, no backflow of infusate was observed along the catheter track to more dorsal cortical ar- eas in any of the animals. The method for calculating tissue volume of distribution is shown in Fig. 3A and B. The volume of distribution in the brain parenchyma obtained for the right substantia nigra corresponds to infusion of $5 \mathrm{mM}$ gadopentetate dimeglumine at a constant rate of $0.1 \mu \mathrm{l} / \mathrm{min}$ over 14 days (Fig. 3C). The volume of distribution in the brain parenchyma obtained for the left substantia nigra corresponds to infusion of $5 \mathrm{mM}$ gadopentetate dimeglumine at a constant rate of $0.1 \mu \mathrm{l} / \mathrm{min}$ over the first 7 days, followed by continued infusion at a constant rate of $0.2 \mu \mathrm{l} / \mathrm{min}$ over the next 7 days (Fig. 3D). As shown in Fig. $3 \mathrm{E}$, the volume of distribution achieved in the midbrain region with the infusate delivered at a maximum rate of 0.2 $\mu \mathrm{l} / \mathrm{min}$ into the left substantia nigra $\left(2052 \pm 168 \mathrm{~mm}^{3}\right)$ was significantly greater than that solely achieved at a constant rate of $0.1 \mu \mathrm{l} / \mathrm{min}$ into the right substantia nigra (1225 \pm $273 \mathrm{~mm}^{3}$ ) by nearly 2 -fold (i.e., 1.9 -fold \pm 0.4 -fold). An MRI taken midinfusion (i.e., 7 days after infusion at 0.1 $\mu \mathrm{l} / \mathrm{min}$ ) for each catheter showed the volume of distribution for the right substantia nigra to be $1116 \pm 158 \mathrm{~mm}^{3}$ and the volume of distribution for the left substantia nigra to be $1214 \pm 177 \mathrm{~mm}^{3}$. These volumes were comparable to the infusate volume of distribution achieved after 14 days of constant infusion at a rate of $0.1 \mu \mathrm{l} / \mathrm{min}$ in the right substantia nigra $\left(1,225 \pm 273 \mathrm{~mm}^{3}\right)$, suggesting that steady state was reached within the first 7 days at a rate of $0.1 \mu \mathrm{l} /$ $\min$.

\section{Morphological Evaluations}

There were no gross findings noted at necropsy. Microscopic evaluations were conducted on full coronal sections including at least 3-4 midbrain sections that spanned the delivery catheter site of infusion. In addition, 1 section from the striatum stained for TH (as a marker for dopaminergic neuron processes) was included in the analysis to further assess potential substantia nigra injury.

Microscopic evaluations indicated that the catheter tip was placed in or near the pars compacta region of the 


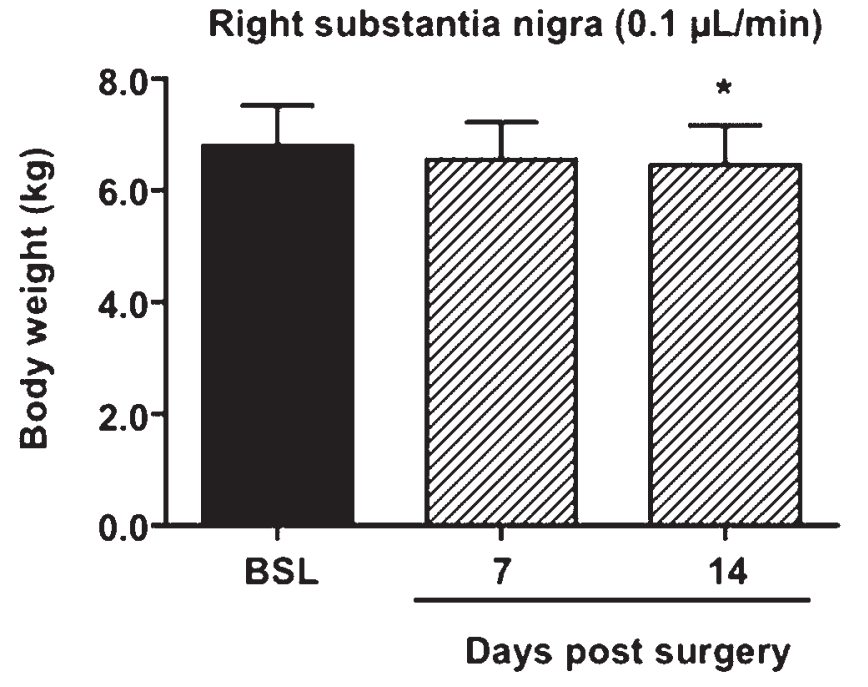

Left substantia nigra $(0.2 \mu \mathrm{L} / \mathrm{min})$

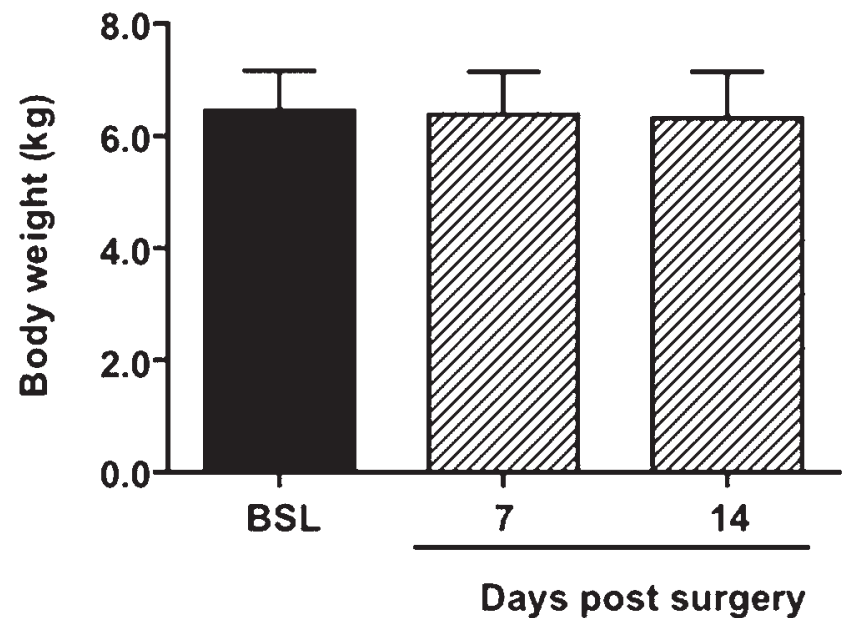

FIG. 2. Body weight measurements. A slight decline in body weight values was observed over time with the infusate delivered at a constant rate of $0.1 \mu \mathrm{l} / \mathrm{min}$ (upper) but not at a maximum rate of $0.2 \mu \mathrm{l} / \mathrm{min}$ (lower) versus presurgical baseline values. ${ }^{*} p<0.05$ versus baseline (BSL), 1-way ANOVA for repeated measures and Dunnett's post hoc test.

right or left substantia nigra in all animals. Key histology assessments reported in Table 2 are further described in the sections below. As indicated in Table 2, the amount of blood, hemosiderin (blood breakdown pigment), and macrophages at the catheter track were variable between animals, as is expected with an intraparenchymal catheter. Blood or hemosiderin at the catheter track was not excessive in any animal. There was no difference between the right and left midbrain region with respect to changes due to local trauma from the catheter insertion. There was no evidence of infection at any of the catheter sites. There was no difference between the 2 infusion rates with respect to changes indicating inflammation, including microglial reactions. Perivascular infiltrates of mononuclear cells (lymphocytes and macrophages), infiltrates of eosinophils (eosinophils are relatively common at the site of a foreign body such as a catheter), and neovascularization (new or prominent blood vessels, a common change at the site of nearly any inflammatory process) were essentially the same between the 2 infusion rates.

As illustrated in Fig. 4, nigral tissue loss was very circumscribed (less than $1 \mathrm{~mm}$ in diameter) and limited to tissue surrounding the catheter tip. The size and/or shape of the site of tissue loss may have been slightly altered during processing, as antemortem tissue was presumed to be closely applied to the edge of the catheter. Mild to moderate microglial reactions were observed at most of the catheter track sites. Microglial cells were activated due to phagocytosis of cellular debris in response to tissue damage. There was no discernible difference regarding the microglial reaction (as determined using the HLA-DR stain) at the sites receiving the 2 different infusion rates. A slight increase of extracellular fluid and a slightly increased astrocyte reaction was present on the side that received the $0.2-\mu \mathrm{l} / \mathrm{min}$ infusion (equivalent to $288 \mu \mathrm{l} / \mathrm{day}$ ) as compared with the $0.1-\mu \mathrm{l} / \mathrm{min}$ infusion (equivalent to $144 \mu \mathrm{l} /$ day). This slight increase of extracellular fluid was most easily visualized on the slides stained with the Nissl stain. The increase of extracellular fluid was seen as an increased distance, in general, between the cells around the area of the infusion on the side that received $288 \mu \mathrm{l} /$ day. There was no detectable decrease of $\mathrm{TH}$ staining in the striatum in any of the sections, and no detectable difference between the 2 infusion rates in any of the animals. Finally, there was no detectable necrosis of neurons in the pars compacta region of the substantia nigra. Additional brain tissues that were also present on the full coronal sections and examined included the meninges, cerebrum/ neocortex, ventricular system, and limbic system/hippocampus. No evidence of pathology was observed in these brain regions/systems.

\section{Discussion}

This investigational study was conducted in 3 female rhesus monkeys to evaluate the tolerability of continuous intranigral infusion of $5 \mathrm{mM}$ gadopentetate dimeglumine delivered via a needle-tip catheter and a commercially available, programmable pump system at a maximum flow rate of either $0.1 \mu \mathrm{l} / \mathrm{min}(144 \mu \mathrm{l} /$ day) to the right substantia nigra or $0.2 \mu \mathrm{l} / \mathrm{min}(288 \mu \mathrm{l} /$ day $)$ to the left substantia nigra. Each implant cycle lasted 14 days. Gadopentetate dimeglumine, an MRI contrast agent, was used to noninvasively assess catheter patency and volume of distribution in the midbrain region using MRI techniques. Flow-rate tolerability was assessed by monitoring clinical observations, body weights, and a microscopic examination of the striatum and midbrain region using histological staining techniques.

There were no indications of motor dysfunction or other complications as noted in clinical observations, indicating that the surgical procedures associated with placement of the delivery system and subsequent continuous infusion to the midbrain region over a 14-day period per catheter was well tolerated by all animals. A change in body weight values was recorded during the study: a $5.2 \% \pm 0.6 \%$ decline after the first infusion and a $2.4 \% \pm 2.2 \%$ decline after the second infusion cycle. We attribute this decline in body weight values measured during the course of the study to 

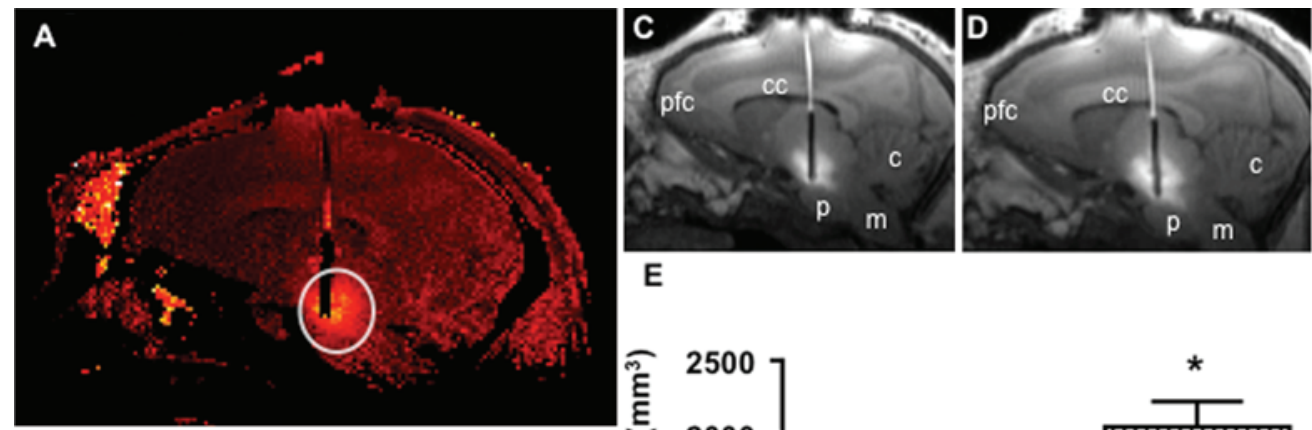

$\mathrm{E}$
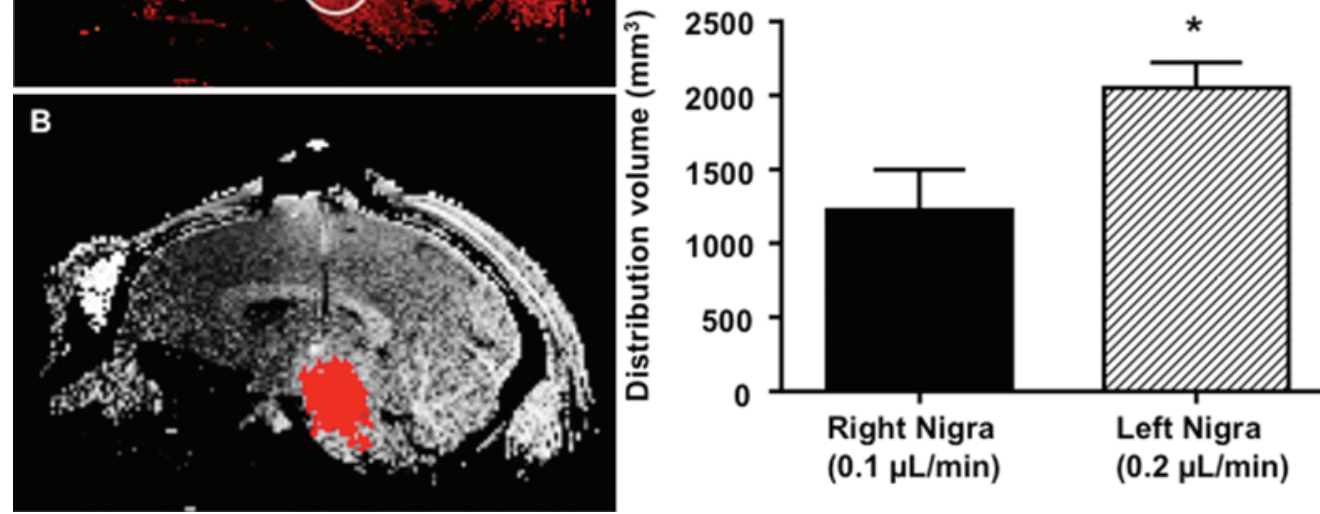

FIG. 3. Infusate tissue volume of distribution. The distortion in the images at the top of the scalp arises from the presence of a metal anchor used to secure the implanted catheter. The apparent dimension of the catheter tip is amplified as a result of partial volume effects and distortion due to the presence of the platinum iridium needle. The method for calculating tissue volume of distribution is shown in the left panels. A: One sagittal slice of a representative calculated 1/T1 image derived from a pair of T1weighted images. B: The same slice as in the panel A image is shown to illustrate the region selected for inclusion in the volume estimate. C: Sagittal T1-weighted image showing infusate distribution at a rate of $0.1 \mu \mathrm{l} / \mathrm{min}$. D: Sagittal T1-weighted image showing infusate distribution at a rate of $0.2 \mu \mathrm{l} / \mathrm{min}$. E: The volume of distribution achieved in the left midbrain region with the infusate delivered at a maximum rate of $0.2 \mu \mathrm{l} / \mathrm{min}$ was nearly 2 -fold greater than that achieved in the right midbrain region with the infusate delivered at a constant rate of $0.1 \mu \mathrm{l} / \mathrm{min}$. ${ }^{*} p<0.05$ versus right substantia nigra, 1 -tailed unpaired t-test. $c=$ cerebellum; $\mathrm{cc}=$ corpus callosum; $\mathrm{m}=$ medulla; $\mathrm{p}=$ pons; $\mathrm{pfc}=$ prefrontal cortex .

a transient decrease in food consumption, resulting from the multiple surgical and MRI procedures requiring general anesthesia. However, we cannot rule out a clinical effect from the infusion itself. This observation is consistent with that of others reporting an average $3.4 \%$ body weight decline in rhesus macaques receiving gadolinium-diethylenetriamine pentaacetic acid via a catheter sequentially placed into the left and right putamen for a period of 14 days per infusion cycle. ${ }^{9}$

Evaluation of postsurgical MRI indicated that all catheters remained patent throughout the study, as evidenced by the presence of a high-contrast signal from the infusate in midbrain tissue surrounding the catheter tip. No backflow of infusate to more dorsal cortical areas was observed

TABLE 2. Histopathological findings*

\begin{tabular}{|c|c|c|c|c|c|c|c|c|}
\hline \multirow[b]{2}{*}{ Diagnosis } & \multicolumn{4}{|c|}{ Right SN $(0.1 \mu l / \mathrm{min})$} & \multicolumn{4}{|c|}{ Left SN $(0.2 \mu \mathrm{l} / \mathrm{min})$} \\
\hline & NHP 1 & NHP 2 & NHP 3 & Avg & NHP 1 & NHP 2 & NHP 3 & Avg \\
\hline $\mathrm{CT}$, hemosiderin & 3 & 1 & 1 & 1.67 & 1 & 0 & 0 & 0.33 \\
\hline CT, blood & 0 & 2 & 0 & 0.67 & 0 & 0 & 1 & 0.33 \\
\hline CT, macrophages & 4 & 3 & 3 & 3.33 & 4 & 0 & 1 & 1.67 \\
\hline $\mathrm{CT}$, perivascular infiltrates/mononuclear cells & 1 & 0 & 1 & 0.67 & 1 & 0 & 0 & 0.33 \\
\hline $\mathrm{CT}$, neovascularization & 2 & 1 & 1 & 1.33 & 1 & 1 & 0 & 0.67 \\
\hline CT, multinucleated giant cells & 0 & 0 & 0 & 0.00 & 2 & 0 & 0 & 0.67 \\
\hline CT, inflitrates, eosinophils & 1 & 0 & 0 & 0.33 & 1 & 0 & 0 & 0.33 \\
\hline $\mathrm{CT}$, astrocytosis & 2 & 2 & 2 & 2.00 & 3 & 3 & 2 & 2.67 \\
\hline CT, microgliosis & 3 & 4 & 3 & 3.33 & 4 & 3 & 2 & 3.00 \\
\hline FJB, positive neurites & 1 & 1 & 1 & 1.00 & 1 & 1 & 1 & 1.00 \\
\hline FJB, positive neurons & 1 & 0 & 1 & 0.67 & 1 & 0 & 1 & 0.67 \\
\hline
\end{tabular}

$\mathrm{Avg}=$ average CT = catheter track; FJB = Fluoro-Jade B; NHP = nonhuman primate; $\mathrm{SN}=$ substantia nigra.

* Microscopic findings were graded according to a 5 -point semiquantitative grading scale in order of increasing severity, where $5=$ severe pathology (see Table 1). 

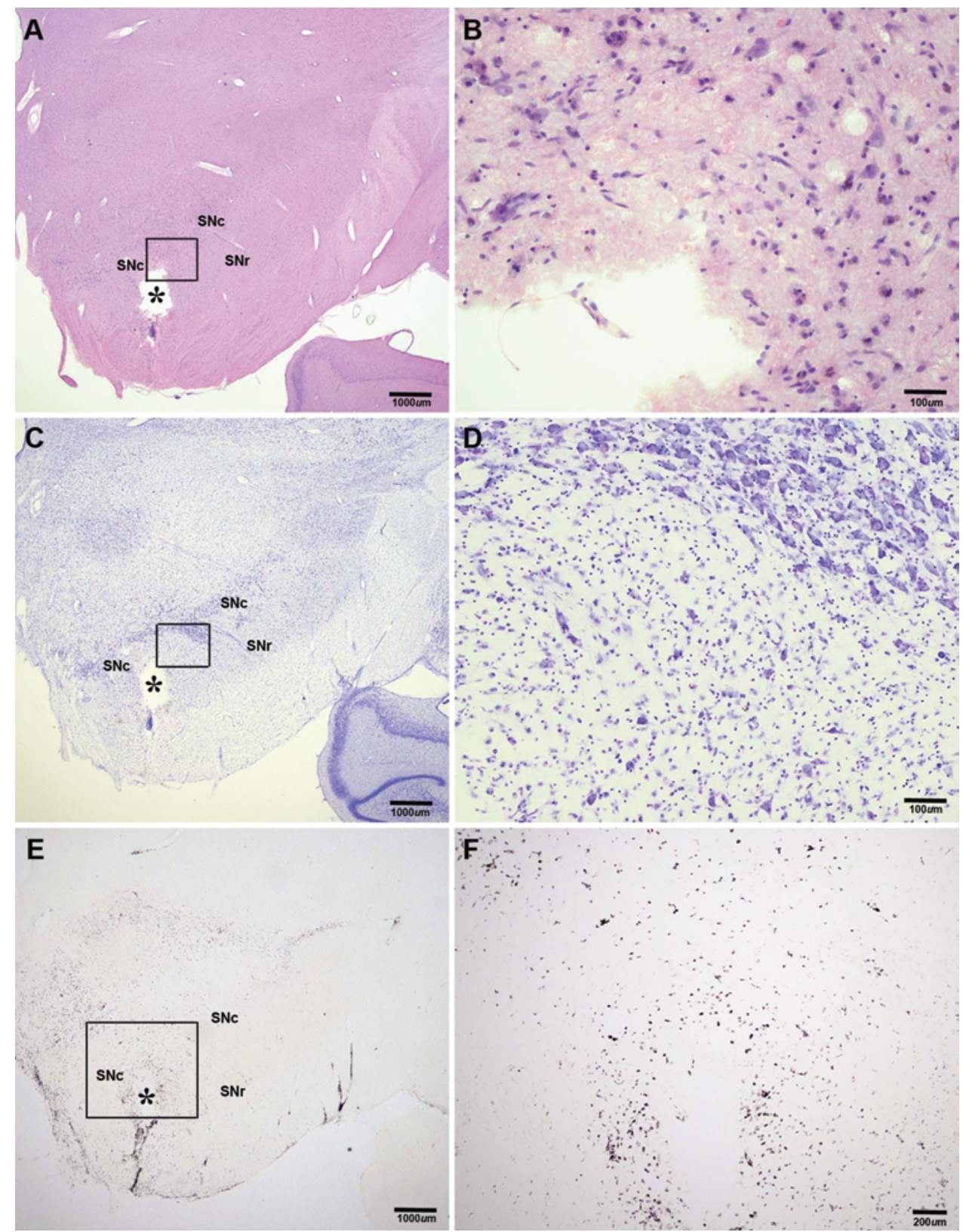

FIG. 4. Micrographs indicating neuropathology. Coronal tissue sections stained for $H \& E(A$ and $B)$, Nissl (C and D), and human leukocyte antigen ( $E$ and $\mathbf{F}$ ) are shown at the level of the left substantia nigra from an animal receiving the infusate at a rate of up to $0.2 \mu \mathrm{l} / \mathrm{min}$. The site of the catheter tip is indicated with an asterisk. All catheter tips were placed in the substantia nigra region in all study animals. Other than very circumscribed tissue loss at the catheter tip $(<1 \mathrm{~mm}$ in diameter), there was no detectable loss of pars compacta neurons and no evidence of infection at any of the 6 catheter sites. Note that the size and/or shape of the site of tissue loss may have been slightly altered during processing, as antemortem tissue was presumed to be closely applied to the edge of the catheter. Mild to moderate microglial reactions were observed at most of the catheter track sites. For the higher magnification inset panels from the left column, bar $=1000 \mu \mathrm{m}(\times 1)$ in $\mathrm{A}, \mathrm{C}$, and $\mathrm{E} . \mathrm{Bar}=100 \mu \mathrm{m}(\times 10)$ in $\mathrm{B}$ and D and $200 \mu \mathrm{m}(\times 4)$ in $\mathrm{F}$. $\mathrm{SNc}=$ substantia nigra pars compacta, $\mathrm{SNr}=$ substantia nigra pars reticulate; $u \mathrm{~m}=$ micrometers.

along the catheter track in any of the animals. This is in line with data published by Fan and colleagues, ${ }^{9}$ who used a flow rate of $0.3 \mu \mathrm{l} / \mathrm{min}$ for intraputamenal infusions without any occurrence of backflow to the cortex. Volumes of distribution averaging $1,225 \pm 273 \mathrm{~mm}^{3}$ were achieved in the right midbrain region with infusion of gadopentetate dimeglumine delivered at a constant rate of $0.1 \mu \mathrm{l} / \mathrm{min}$. Consistent with volume of distribution being proportional to the increase in infusion flow rate as reported by others, ${ }^{9}$ postsurgical MRI evaluation indicated that the volume of distribution achieved in the left midbrain region with gadopentetate dimeglumine infused at a maximum rate of 0.2 $\mu \mathrm{l} / \mathrm{min}\left(2052 \pm 168 \mathrm{~mm}^{3}\right)$ was greater than that achieved in the right midbrain region at a constant rate of $0.1 \mu \mathrm{l} / \mathrm{min}$ $\left(1225 \pm 273 \mathrm{~mm}^{3}\right)$ by nearly 2 -fold. A recent voxel-based morphometry and volumetric study conducted in human 
subjects using a 3T Siemens Trio MR scanner reported the volume (mean \pm standard deviation) of the human substantia nigra to range from $484 \pm 112 \mathrm{~mm}^{3}$ in PD patients $(\mathrm{n}=$ 20) to $556 \pm 144 \mathrm{~mm}^{3}$ in normal age-matched controls (n $=19) \cdot{ }^{16}$ By comparison, Pakkenberg and colleagues ${ }^{17}$ have reported the total volumetric measurements of the subtantia nigra in a group of 16 female rhesus monkeys to range from 50 to $110 \mathrm{~mm}^{3}$, supporting that the 2 flow rates we tested (even at the lower rate of $0.1 \mu \mathrm{l} / \mathrm{min}$ ) can result in sufficient infusate coverage of the rhesus, and possibly the human, midbrain region, assuming correct catheter placement. One limitation of our study is that the volume of distribution achieved with gadopentetate dimeglumine may be different for other, more complex test molecules. Additional studies would be required to determine how the addition of larger trophic factor molecules or siRNA constructs (or others) to the infusate would affect volume of tissue distribution.

Tissue reaction to the catheter and the infusate was equal to or less than what would be expected to occur when a catheter is placed in any brain location. There was no evidence of infection at any of the catheter sites. Other than very circumscribed tissue loss at the catheter tip (less than $1 \mathrm{~mm}$ in diameter), there was no detectable loss of pars compacta neurons supporting that continuous intranigral infusion using our needle-tip catheter was achieved without causing marked pathology. Just as important, there was no detectable decrease of TH staining in the striatum. The striatum contains numerous, rather diffusely arranged, dopaminergic synaptic terminals and fibers traceable to cell bodies in the pars compacta region of the substantia nigra. TH staining in the striatum is often the most sensitive morphological indicator of a decrease of dopaminergic neurons in the pars compacta region. Overall, continuous delivery of the infusate to the midbrain region of the brainstem did not result in any morphological changes that would be expected to have adverse biological consequences. Our data are in accordance with those obtained by Bartus and colleagues, showing that delivery of viral vectors encoding for the trophic factor neurturin directly into the substantia nigra can be safely achieved in both nonhuman primates and parkinsonian patients. ${ }^{2,3}$ Toxicity associated with continuous drug delivery to the brain parenchyma is likely to be highly dependent on the nature of the agent being infused. Further studies would be required to determine whether the addition of a larger molecule or biological to the infusate over longer delivery periods might exacerbate the associated morphological changes reported here.

\section{Conclusions}

Our data support that intranigral infusion using a programmable pump-and-catheter system does not cause observable behavioral deficits or marked pathology in rhesus macaques and lay the foundation for using this approach to deliver therapeutic compounds to treat PD patients or patients with related degenerative diseases that would benefit from direct drug delivery into the substantia nigra or other brainstem areas.

\section{References}

1. Ai Y, Markesbery W, Zhang Z, Grondin R, Elseberry D, Gerhardt GA, et al: Intraputamenal infusion of GDNF in aged rhesus monkeys: distribution and dopaminergic effects. J Comp Neurol 461:250-261, 2003

2. Bartus RT, Baumann TL, Siffert J, Herzog CD, Alterman R, Boulis N, et al: Safety/feasibility of targeting the substantia nigra with AAV2-neurturin in Parkinson patients. Neurology 80:1698-1701, 2013

3. Bartus RT, Brown L, Wilson A, Kruegel B, Siffert J, Johnson EM Jr, et al: Properly scaled and targeted AAV2-NRTN (neurturin) to the substantia nigra is safe, effective and causes no weight loss: support for nigral targeting in Parkinson's disease. Neurobiol Dis 44:38-52, 2011

4. Bartus RT, Herzog CD, Chu Y, Wilson A, Brown L, Siffert $\mathrm{J}$, et al: Bioactivity of AAV2-neurturin gene therapy (CERE120): differences between Parkinson's disease and nonhuman primate brains. Mov Disord 26:27-36, 2011

5. Bartus RT, Kordower JH, Johnson EM Jr, Brown L, Kruegel $\mathrm{BR}, \mathrm{Chu} \mathrm{Y}$, et al: Post-mortem assessment of the short and long-term effects of the trophic factor neurturin in patients with $\alpha$-synucleinopathies. Neurobiol Dis 78:162-171, 2015

6. Braak H, Del Tredici K, Rüb U, de Vos RAI, Jansen Steur ENH, Braak E: Staging of brain pathology related to sporadic Parkinson's disease. Neurobiol Aging 24:197-211, 2003

7. Committee for the Update of the Guide for the Care and Use of Laboratory Animals: Guide for the Care and Use of Laboratory Animals. Washington, DC: National Academies Press, 2011

8. Deoni SC, Peters TM, Rutt BK: High-resolution T1 and T2 mapping of the brain in a clinically acceptable time with DESPOT1 and DESPOT2. Magn Reson Med 53:237-241, 2005

9. Fan X, Nelson BD, Ai Y, Stiles DK, Gash DM, Hardy PA, et al: Continuous intraputamenal convection-enhanced delivery in adult rhesus macaques. J Neurosurg 123:1569-1577, 2015

10. Gill SS, Patel NK, Hotton GR, O'Sullivan K, McCarter R, Bunnage M, et al: Direct brain infusion of glial cell linederived neurotrophic factor in Parkinson disease. Nat Med 9:589-595, 2003

11. Grondin R, Zhang Z, Elsberry DD, Gerhardt GA, Gash DM: Chronic intracerebral delivery of trophic factors via a programmable pump as a treatment for parkinsonism. Methods Mol Med 62:257-267, 2001

12. Lang AE, Gill S, Patel NK, Lozano A, Nutt JG, Penn R, et al: Randomized controlled trial of intraputamenal glial cell linederived neurotrophic factor infusion in Parkinson disease. Ann Neurol 59:459-466, 2006

13. Lee VM, Trojanowski JQ: Mechanisms of Parkinson's disease linked to pathological alpha-synuclein: new targets for drug discovery. Neuron 52:33-38, 2006

14. Lees AJ, Tolosa E, Olanow CW: Four pioneers of L-dopa treatment: Arvid Carlsson, Oleh Hornykiewicz, George Cotzias, and Melvin Yahr. Mov Disord 30:19-36, 2015

15. McCormack AL, Mak SK, Henderson JM, Bumcrot D, Farrer MJ, Di Monte DA: Alpha-synuclein suppression by targeted small interfering RNA in the primate substantia nigra. PLoS One 5:e12122, 2010

16. Menke RAL, Szewczyk-Krolikowski K, Jbabdi S, Jenkinson M, Talbot K, Mackay CE, et al: Comprehensive morphometry of subcortical grey matter structures in early-stage Parkinson's disease. Hum Brain Mapp 35:1681-1690, 2014

17. Pakkenberg H, Andersen BB, Burns RS, Pakkenberg B: A stereological study of substantia nigra in young and old rhesus monkeys. Brain Res 693:201-206, 1995

18. Salvatore MF, Ai Y, Fischer B, Zhang AM, Grondin RC, Zhang Z, et al: Point source concentration of GDNF may explain failure of phase II clinical trial. Exp Neurol 202:497505,2006 
19. Slevin JT, Gerhardt GA, Smith CD, Gash DM, Kryscio $\mathrm{R}$, Young B: Improvement of bilateral motor functions in patients with Parkinson disease through the unilateral intraputaminal infusion of glial cell line-derived neurotrophic factor. J Neurosurg 102:216-222, 2005

20. Stiles DK, Zhang Z, Ge P, Nelson B, Grondin R, Ai Y, et al: Widespread suppression of huntingtin with convection-enhanced delivery of siRNA. Exp Neurol 233:463-471, 2012

21. Visanji NP, Brooks PL, Hazrati LN, Lang AE: The prion hypothesis in Parkinson's disease: Braak to the future. Acta Neuropathol Commun 1:2, 2013

\section{Disclosures}

Financial support for this study was provided to the University of Kentucky (R.G.) by Alnylam Pharmaceuticals Inc., with funding received from the Michael J. Fox Foundation for Parkinson's Research via a competitive LEAPS award. The hardware and software associated with the delivery system were provided by Medtronic Inc. Mr. Nelson is a current employee of Medtronic Inc. Dr. Lemmon was an employee of Medtronic Inc. at the time the work was completed. Dr. Bumcrot is a former employee of Alnylam Pharmaceuticals and a current employee of Editas Medicine Inc.

\section{Author Contributions}

Conception and design: Grondin, Bumcrot, Gash, Gerhardt, Zhang. Acquisition of data: Ai, Hardy, Butt, Zhang. Analysis and interpretation of data: Grondin, Hardy, Butt. Drafting the article: Grondin. Critically revising the article: Ai, Hardy, Butt, Nelson, Lemmon, Bumcrot, Gash, Gerhardt, Zhang. Reviewed submitted version of manuscript: Grondin, Ai, Hardy, Butt, Nelson, Lemmon, Bumcrot, Gash, Gerhardt, Zhang. Approved the final version of the manuscript on behalf of all authors: Grondin. Statistical analysis: Grondin. Administrative/technical/material support: Nelson, Lemmon. Study supervision: Grondin.

\section{Correspondence}

Richard Grondin, Department of Anatomy \& Neurobiology, University of Kentucky Medical Center, 800 Rose St., Rm. MN210 Medical Bldg., Lexington, KY 40536-0298. email: rcgron0@uky.edu. 